\title{
Simultaneous Kidney-Pancreas Transplantation With an Original "Transverse Pancreas" Technique: Initial 9 Years' Experience With 56 Cases
}

\author{
J. Paulino ${ }^{a, *}$, A. Martins ${ }^{a}$, E. Vigia ${ }^{a}$, P. Marcelino ${ }^{b}$, A.M. Nobre ${ }^{a}$, L. Bicho ${ }^{a}$, E. Filipe ${ }^{a}$, and E. Barroso ${ }^{a}$ \\ ${ }^{a}$ Centro Hepatobiliopancreático e de Transplantação, Centro Hospitalar de Lisboa Central, Universidade Nova de Lisboa, Lisboa, \\ Portugal; and ' ${ }^{\mathrm{C}} \mathrm{CEDOC}$, Faculdade de Ciências Médicas, Universidade Nova de Lisboa, Lisboa, Portugal
}

\begin{abstract}
An innovative technique for pancreas transplantation is described. The main aspect consists of the horizontal positioning of the pancreas, which allows a better venous outflow, thus preventing thrombosis and graft loss. The program of pancreas transplantation in this national reference center for pancreatic and liver surgery was started in 2007; the initial results were considered poor, resulting in the loss of half of the grafts due to venous thrombosis. After analyzing the possible causes, this technique was proposed and successfully implemented, reducing the postoperative complications, particularly the problem of venous thrombosis. A detailed description of the new surgical technique is provided. The main clinical and demographic characteristics of the 56 patients who underwent the surgery are analyzed. The incidence of venous thrombosis was $5.3 \%$ ( 3 patients) and graft loss was $3.5 \%$ ( 2 patients). Due to the good results, this technique became the standard surgery for transplantation of the pancreas in our center. The technique proved to be safe and successful. Due to the unique pancreas graft implantation, we called it "transverse pancreas surgery."
\end{abstract}

$\mathbf{S}$ IMULTANEOUS pancreas and kidney transplantation (SPK) is indicated in uremic type 1 insulin-dependent diabetic patients, because end-stage renal disease has ominous prognostic implications in this population: $75 \%$ percent of insulin-dependent diabetic patients do not survive longer than 5 years while receiving dialysis [1].

Pancreas transplantation (PT) remains a technically challenging surgery, mainly due to the unique and complicated blood supply. For this reason, many different surgical techniques have been described to overcome the high rate of surgical complications. The implantation position of the pancreas graft is conditioned by the type of endocrine venous delivery chosen (portal or systemic) and of exocrine drainage (either bladder or enteric). Since 1995, the primary enteric exocrine drainage is described in more than $80 \%$ in the literature, with the pancreas graft in a vertical position in any kind of endocrine drainage [2]. There are also reports of transversal implantation of the pancreas graft in some old series of whole PT using the common iliac vessels [3].

In our center, the PT program was started in 2007, using the vertical position of the pancreas graft. However, the first 8 patients presented a high rate of complications, namely venous thrombosis in 4 patients, which led to pancreas graft loss [4]. To improve outcome of these patients, the surgical technique was modified to an innovative solution that consisted in the horizontal positioning of the pancreas (Fig 1). This technique is described here.

\section{MATERIALS AND METHODS \\ Patients}

Between February 2007 and October 2016, a total of 61 patients underwent a total of 64 PTs, as follows: 59 SPKs, 2 pancreas after kidney transplantations (PAKs), 3 pancreas retransplantations (2 PAKs and 1 SPK). As previously stated, in 8 patients the vertical position of the pancreas graft was used, and in the remaining 56 patients the new approach was used.

Data collected from these 56 recipients included age, sex, duration of diabetes disease, previous coronary intervention, smoking status, time on waiting list, and cold ischemia time. Also the

*Address correspondence to Jorge Paulino, MD, PhD, Centro Hepatobiliopancreático e de Transplantação, Centro Hospitalar de Lisboa Central, Universidade Nova de Lisboa, Rua da Beneficência, 8, 1069-166 Lisboa, Portugal. E-mail: fusilis@gmail.com

$0041-1345 / 17$

http://dx.doi.org/10.1016/j.transproceed.2017.04.015 


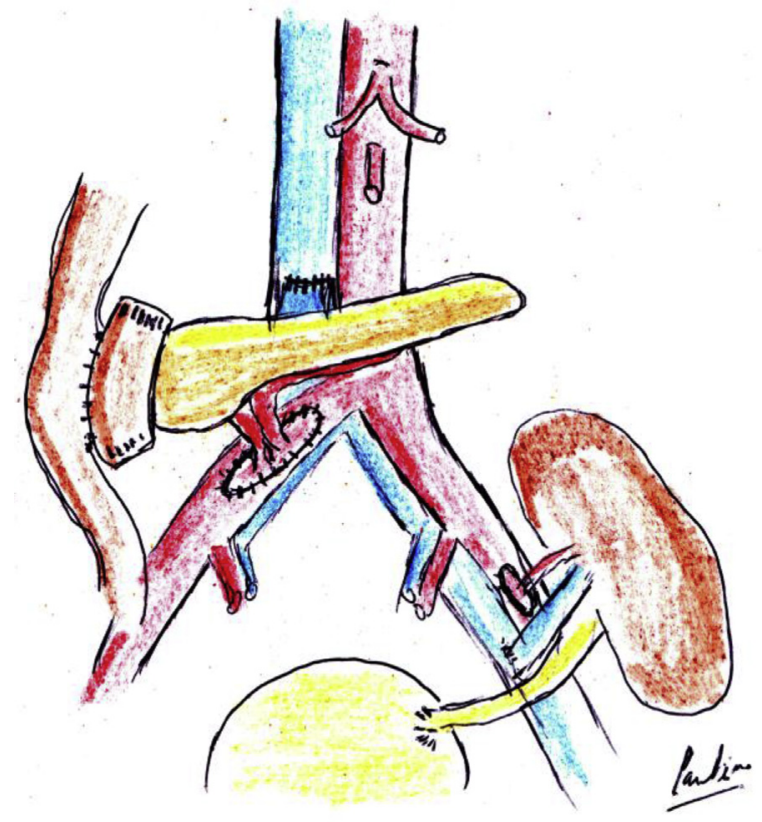

Fig 1. Depiction of pancreas and kidney transplantation, showing vascular and enteric anastomoses, with the "transverse pancreas" technique.

HLA-A + -B mismatch and HLA-DR mismatch were considered. The complications considered were surgical reoperation due to correction of hemostasis, thrombosis, as well as pancreas and kidney graft loss and death occurring in the first 3 months after transplantation. Donor data such as age, sex, body mass index, and cause of death were also collected.

\section{Donor Operation}

All the pancreases were procured en bloc with the liver by the same experienced team [5]. The same technique was always used. All donors were under 50 years old and hemodynamically stable. All donor organs were preserved by Celsior solution $[6,7]$.

The aortic Carrel patch, including the celiac trunk and the superior mesenteric artery (SMA) ostia, was kept with the pancreas graft in all the cases.

\section{Back Table Bench Surgery}

Bench preparation was always performed by the same 2 experienced senior surgeons. In all the cases, the pancreas and liver were procured by the same team, and the aortic patch was kept with the pancreas graft. In 57 cases, the arterial flow through the gastroduodenal artery (GDA) was preserved in the pancreas by separating the liver from the pancreas graft at the level of the proper hepatic artery. In one case, the common hepatic artery and the GDA stumps were ligated.

\section{Recipient Surgery}

A midline incision from the pubis to $3 \mathrm{~cm}$ above the umbilicus was performed. The transverse pancreas technique was accomplished by using 2 Satinsky vascular clamps, and the inferior vena cava (IVC) was totally clamped and an appropriate length venotomy was

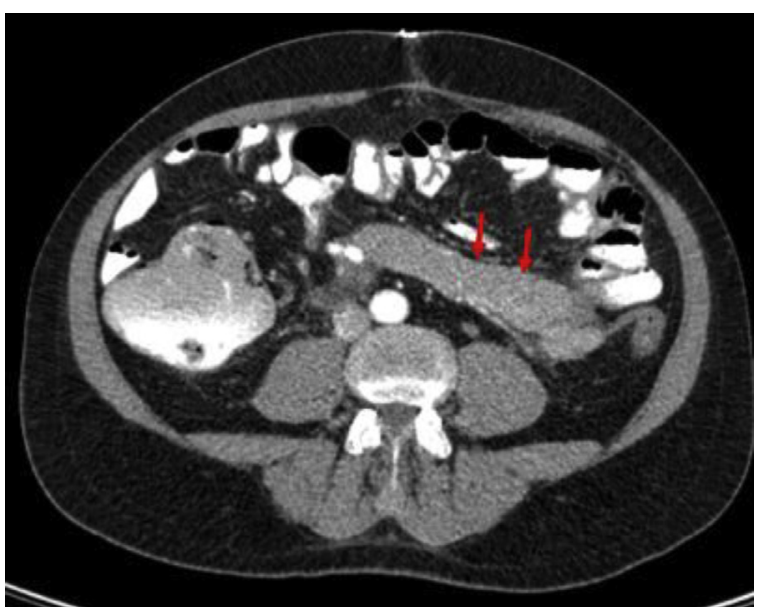

Fig 2. Computed tomography scan 3 weeks after the transplant, confirming the pancreas graft in the "transverse" position (red arrows).

performed transversely to match the donor portal vein diameter (Fig 2). The donor portal vein was then anastomosed end to side to the IVC using 5-0 Prolene (Ethicon, Johnson \& Johnson, Belgium) continuous sutures. Then the fashioned aortic Carrel patch with the SMA and the celiac trunk ostia was anastomosed end to side to the recipient right common iliac artery using 5-0 Prolene continuous sutures (Fig 1). In this technique, the pancreas is emplaced transversely. The procedure was completed by 2-layer hand-sewn side-toside anastomoses between the donor duodenum and an adjacent loop of jejunum using 4-0 Vicryl (Ethicon, Johnson \& Johnson, Belgium) and 4-0 Prolene running sutures.

The kidney graft was implanted after the pancreas in all the cases, retroperitoneally on the left iliac fossa, using the same incision, following the technique described in the literature by Watson et al [8]. A urinary stent was left in place as well as a Foley catheter in all cases.

\section{Immunosuppression}

All the recipients received the quadruple immunosuppressive therapy.

\section{Anticoagulation and Prophylactic Antimicrobial Therapy}

In all patients, anticoagulation and prophylactic antimicrobial therapy consisted of acetylsalicylic acid, starting at day 0; low molecular weight heparin (nadroparin); megalotect; vancomycin fluconazol; and valganciclovir.

\section{RESULTS}

The recipients' and donors' clinical and demographical characteristics are described in Table 1. The overall complications are described in Table 2.

All anastomoses were easy to perform in all the patients, even in those who had a high body mass index. The pancreas graft loss and the kidney graft loss was $7.1 \%$ and $3.5 \%$, respectively. Except in one case (blunt abdominal trauma), all the graft losses, pancreas or kidney, were due to venous thrombosis. In 3 cases, we had to perform an early allograft 
Table 1. General Characteristics of the Recipient and Donor Populations

\begin{tabular}{lc}
\hline \multicolumn{1}{c}{ Parameters } & Result \\
\hline Recipient $(\mathrm{n}=56)$ & $34.4 \pm 6.8$ \\
Age $(\mathrm{y})$, mean \pm SD & $34(60.7 \%)$ \\
Male, $\mathrm{n}(\%)$ & 56 \\
$\mathrm{PT}(\mathrm{n})$ & $51(91.07 \%)$ \\
SPK, $\mathrm{n}(\%)$ & $2(3.57 \%)$ \\
PAK, $\mathrm{n}(\%)$ & $3(5.35 \%)$ \\
PR, $\mathrm{n}(\%)$ & $24.6 \pm 6.3$ \\
BMI $\left(\mathrm{Kg} / \mathrm{m}^{2}\right)$ & $24.7 \pm 6.7$ \\
Diabetes duration at time of transplant $(\mathrm{y})$ & $2(3.57 \%)$ \\
Coronary artery intervention pretransplant, $\mathrm{n}(\%)$ & $6(10.71 \%)$ \\
Smoking status previous or current, $\mathrm{n}(\%)$ & $210 \pm 170$ \\
Time on waiting list (d) & $47(83.9 \%)$ \\
Dialysis pretransplant, $\mathrm{n}(\%)$ & $485 \pm 130$ \\
Cold ischemia time (min) & - \\
HLA-A $+-\mathrm{B}$ mismatch & - \\
HLA-DR mismatch & \\
Donor $(\mathrm{n}=56)$ & $35 \pm 10$ \\
Age $(\mathrm{y})$, mean \pm SD & $39(69.6 \%)$ \\
Male, $\mathrm{n}(\%)$ & 25.49 \\
BMI (kg/m $\left.{ }^{2}\right)$ & $24(42.8 \%)$ \\
Cause of death trauma, $\mathrm{n}(\%)$ & \\
\hline
\end{tabular}

Abbreviations: BMI, body mass index; PAK, pancreas after kidney transplantation; PR, pancreas retransplantation; PT, pancreas transplantation; SD, standard deviation; SPK, simultaneous pancreas kidney transplantation.

pancreatectomy using the technique described in literature $[9,10]$. The 3 patients previously transplanted using the vertical technique were retransplanted using the transverse technique.

The postoperative mortality was $3.5 \%$. One patient developed sepsis after venous thrombosis of both pancreas and kidney grafts, and another developed a postoperative cardiac arrest with anoxic encephalopathy.

We registered 2 reoperations by other causes, one due to small bowel occlusion due to a twist of the duodenojejunal anastomosis and another after blunt abdominal trauma with splenic artery (SA) rupture. We observed one transverse pancreas venous thrombosis early in our experience with this technique when we performed a longitudinal IVC venotomy. The other 2 cases of pancreas graft venous thrombosis occurred simultaneously with renal graft vein thrombosis, revealing other probable causes unrelated with the implantation technique.

Table 2. Complications

\begin{tabular}{lc}
\hline \multicolumn{1}{c}{ Complication } & $\mathrm{n}(\%)$ \\
\hline Reoperation & \\
Total & $10(17.8 \%)$ \\
Hemostasis & $6(10.7 \%)$ \\
Thrombosis & $3(5.3 \%)$ \\
Other & $2(3.5 \%)$ \\
Pancreas graft loss & $4(7.1 \%)$ \\
Kidney graft loss in SPKs & $2(3.5 \%)$ \\
Patient death & $2(3.5 \%)$ \\
\hline
\end{tabular}

Abbreviation: SPK, simultaneous pancreas kidney transplantation.

\section{DISCUSSION}

The results showed that the newly described transverse pancreas technique can be performed safely and successfully in any kind of PT recipient, and it can be considered as a good alternative when performing the systemic endocrine and enteric exocrine drainage. Moreover, with this new technique we can reduce complications and graft loss.

The transverse position of the whole pancreas graft allows an ideal arterial irrigation as well as venous drainage without any tension, by keeping the complete graft untwisted all the time, even when the kidney is implanted on the right side. This fact has been confirmed by our team, not only by the relaparotomies performed but also by the image of the postoperative abdominal computed tomography scans (Fig 2).

We believe, as others have reported, that the IVC is the ideal choice for venous drainage, because slow venous flow has been blamed for pancreas allograft failure [11-14]; the IVC allows the best flow provided the venotomy is transversal and well calibrated. So far, we have not registered any leak of the duodenal stump graft, described in the literature with an incidence of $4 \%$ to $10 \%$ [15-17] and resulting in pancreatic graft loss in most of the cases, mainly in PAK cases that presented more than 3 times increased odds compared with SPK [17]. The surgical technique of the exocrine drainage changed over time in most centers, by using mostly duodenoenterostomy, as described in our center [18]. We were able to keep the Carrel aortic patch with the pancreas graft in all the cases, permitting a safe arterial vascularization without the need to interpose a Y arterial graft anastomosing the SA and SMA stumps, or other anastomoses that include the 3 arteries (SA, SMA, and GDA) in the so-called "triple" arterial reconstruction [19]. Moreover, the vascularization to the GDA was retained in 57 of the $58 \mathrm{PT}$, establishing the continuity with the common hepatic artery with separate stitches of 6-0 Prolene. By doing so, better arterial duodenal irrigation was kept, covering all the possible anatomical variations without the need of further reconstructions [20,21]. The transverse position of the transverse pancreas permits minimum traction on the portal vein and caval anastomosis with a lower rate of thrombosis, as well as a strong untwisted attachment of the Carrel patch. Additionally, it permits plenty of space to stretch the whole pancreas graft, with the tail fitting perfectly toward the left flank always untwisted.

Opposite to the retroperitoneal position, the duodenum of the pancreas graft is easily amenable to the fashioned enteroduodenostomy in every patient, explaining the very low rate of complications in this report, due to this anastomosis.

\section{Limitations}

This new technique was performed in a single transplantation center, so we cannot conclude that this approach is reproducible in other centers. Nonetheless, this technique proved to be safe and secure, which made it the standard 
surgical technique in this center. For all major surgeries, and especially transplantation techniques, the necessary learning curve in essential. However, this center is the national reference center for pancreatic and liver surgery, including transplantation. The kidney transplant program started in 1989 and the liver transplantation program in 1992, so the interdisciplinary team, including dedicated surgeons, acquired proper experience in this field. Because the complications decreased after the introduction of the transverse pancreas surgery, we think that reasons other than the new technique are not likely.

\section{CONCLUSIONS}

The transverse pancreas way of transplanting a whole pancreas graft is a valid alternative to the classic vertical pancreas and is reproducible, safe, and easy to perform in most patients. With this approach, our transplantation center managed to reduce overall complications.

\section{REFERENCES}

[1] Dinckan A, Aliosmanoglu I, Kocak H, Mesci A, Altunbas A, Gurkan A. The impact of method on kidney graft and patient survival in kidney-pancreas transplantations for type I diabetes mellitus. Int Surg 2015;100:137-41.

[2] Gruessner AC, Sutherland DE. Pancreas transplant outcomes for United States (US) and non-US cases as reported to the United Network for Organ Sharing (UNOS) and the International Pancreas Transplant Registry (IPTR) as of May 2003. Clin Transplant 2003;3:21-51.

[3] Sutherland DE, Goetz FC, Najarian JS. One hundred pancreas transplants at a single institution. Ann Surg 1984;200:414-40.

[4] Stratta RJ, Taylor RJ, Gill IS. Pancreas transplantation: a managed cure approach to diabetes. Curr Probl Surg 1996;33:709-808.

[5] Wunderlich H, Brockmann JG, Voigh R. DTG procurement guidelines in heart beating donors. Transpl Int 2011;24:733-57.

[6] Manrique A, Jimenez C, Herrero ML, Meneu JC, Abradelo M, Moreno A, et al. Pancreas preservation with the University of Wisconsin versus Celsior solutions. Transplant Proc 2006;38:2582-4.

[7] Latchana N, Peck JR, Whitson BA, Henry ML, Elkhammas EA, Black SM. Preservation solutions used during abdominal transplantation: current status and outcomes. World J Transplant 2015;5:154-64.
[8] Watson CJE, Friend PJ. Surgical Techniques of Kidney Transplantation, in Kidney Transplantation - Principles and Practice. In: Morris P, Knechtle S, editors. 7th Ed. Elsevier Saunders; 2013.

[9] Fridell JA, Mangus RS, Mull AB, Taber TE, Sanders CE, Slisher RC, et al. Early reexploration for suspected thrombosis after pancreas transplantation. Transplantation 2011;91:902-7.

[10] Hollinger EF, Powelson JA, Mangus RS, Kazimi MM, Taber TE, Goble ML, et al. Immediate retransplantation for pancreas allograft thrombosis. Am J Transplant 2009;9:740-5.

[11] Ciancio G, Cespedes M, Olson L, Miller J, Burke GW. Partial venous thrombosis of the pancreatic allografts after simultaneous pancreas-kidney transplantation. Clin Transplant 2000;14: 464-71.

[12] Laftavi MR, Pankewycz O, Kohli R, Feng L, Said M, Sharma R, et al. Short and long-term outcomes of systemic drainage to IVC: a new technique for pancreas transplantation. Transplant Proc 2014;46:1900-4.

[13] Muthusamy AS, Giangrande PL, Friend PJ. Pancreas allograft thrombosis. Transplantation 2010;90:705-7.

[14] Horneland R, Paulsen V, Lindahl JP, Grzyb K, Eide TJ, Lundin K, et al. Pancreas transplantation with enteroanastomosis to native duodenum poses technical challenges-but offers improved endoscopic access for scheduled biopsies and therapeutic interventions. Am J Transplant 2015;15:242-50.

[15] Manrique A, Jimenez C, Lopez RM, Cambra F, Morales JM, Andres A, et al. Relaparotomy after pancreas transplantation: causes and outcomes. Transplant Proc 2009;41: 2472-4.

[16] Reddy KS, Stratta RJ, Shokouh-Amiri MH, Alloway R, Egidi MF, Gaber AO. Surgical complications after pancreas transplantation with portal-enteric drainage. J Am Coll Surg 1999;189:305-13.

[17] Spetzler VN, Goldaracena N, Marquez MA, Singh SK, Norgate A, McGilvray ID, et al. Duodenal leaks after pancreas transplantation with enteric drainage-characteristics and risk factors. Transpl Int 2015;28:720-8.

[18] Monroy-Cuadros M, Salazar A, Yilmaz S, McLaughlin K. Bladder vs enteric drainage in simultaneous pancreas-kidney transplantation. Neph Dial Transplant 2006;21:483-7.

[19] Socci C, Orsenigo E, Zuber V, Caldara R, Castoldi R, Parolini D, et al. Triple arterial reconstruction improves vascularization of whole pancreas for transplantation. Transplant Proc 2006;38:1158-9.

[20] Nghiem DD. Revascularization of the gastroepiploic artery in pancreas transplant. Transpl Int 2008;21:774-7.

[21] Boggi U, Amorese G, Marchetti P. Surgical techniques for pancreas transplantation. Curr Opin Organ Transplant 2010;15: $102-11$. 